\title{
Physical Evaluation of an Anthropometric Shape Model of the Human Scalp
}

\author{
Jochen VLEUGELS ${ }^{* 1}$, DaniëI LACKO ${ }^{1,2}$, Guido DE BRUYNE ${ }^{1}$, \\ Toon HUYSMANS ${ }^{2}$, Stijn VERWULGEN ${ }^{1}$ \\ ${ }^{1}$ Product Development, Faculty of Design Sciences, University of Antwerp, Antwerp, Belgium; \\ ${ }^{2}$ iMinds-Vision Lab, Department of Physics, Faculty of Science, Univ. of Antwerp, Antwerp, Belgium
}

DOI: 10.15221/15.161 http://dx.doi.org/10.15221/15.161

\begin{abstract}
Former research has shown that statistical shape models of the human head can be deduced from MRI scans and that 100 models from a random selection suffices to predict any other head shape within the target population (Western adults between 20 and 40 years old). Representativeness was however only verified theoretically using repeated random sub-sampling validation, that is, with respect to other MRI scans from the same database [http://www.loni.usc.edu/ICBM/]. In order to verify the representativeness and accuracy of the statistical shape model for the target population, a study was performed to compare the actual scalp shape of 14 participants with their predicted scalp shape. In this study, all of the participants were measured in several ways and the prediction of the statistical shape model was compared to the actual 3D shape. Moreover, the accuracy of the prediction of an individual's head by the statistical shape model based on only four current and easy to measure anthropometric values was calculated. It was found that the statistical shape model's prediction of a real person's head scalp based on the anthropometric values: head length, head width, head circumference and the arc length over the width of the head, was accurate up to $2.12 \mathrm{~mm}$ where the theoretical verification obtained an accuracy of $1.6 \mathrm{~mm}$.
\end{abstract}

Keywords: Statistical shape model, anthropometry, industrial design, physical verification, human head.
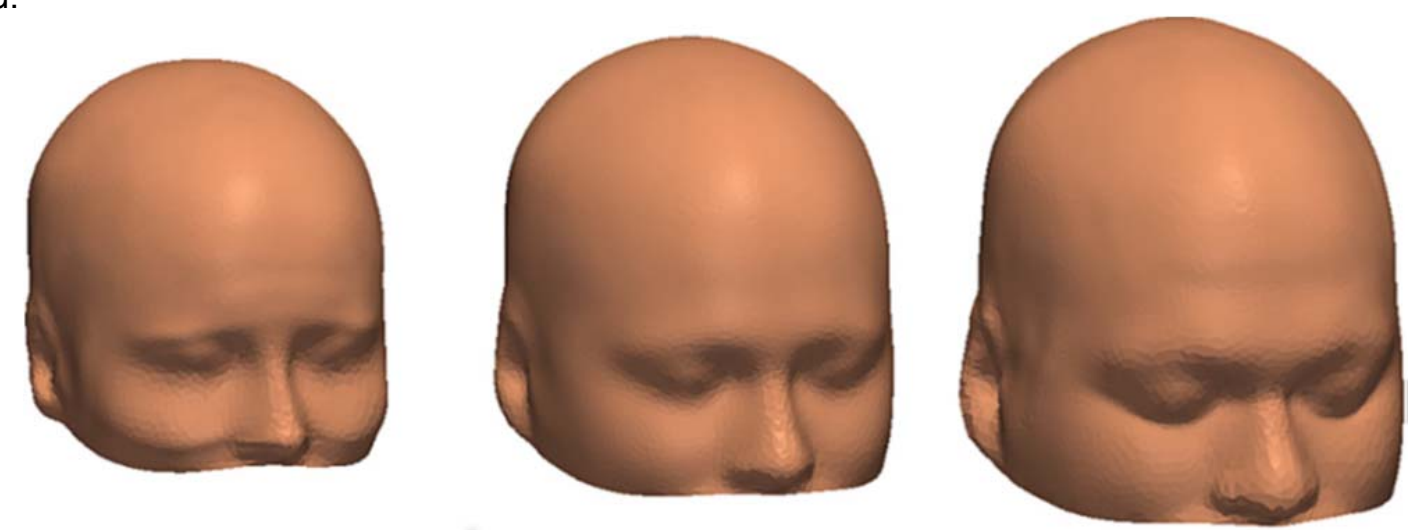

Fig 1: Examples of three generated shapes for a small, an average and a large head sample.

\section{Introduction}

Statistical shape models of the human head are becoming more and more available for the purposes of product development [1]. They supply a 3D template to design products on, can serve as primitives for parametric designs, but can also supply a model to verify designs digitally in the early phase of product development, with regard to e.g. fit and ergonomics. Thereby resource consuming physical verifications might be omitted, resulting in faster development and earlier market introduction. Thus accuracy and representability for the target population are key features as product developers come to rely on these models more and more often. Not only for the design itself but also for methods to predict an individual's head shape based on a series of convenient one, two and three-dimensional measurements shape models are useful for parametrized design in a mass-customization production [2], for constructions of true 3D manikins [3] and to assign an individual to its best fitting product size without a trial-and-error fitting.

\footnotetext{
*Jochen.vleugels@uantwerp.be; www.uantwerp.be
} 


\section{Introduction}

Statistical shape models of the human head are becoming more and more available for the purposes of product development [1]. They supply a 3D template to design products on, can serve as primitives for parametric designs, but can also supply a model to verify designs digitally in the early phase of product development, with regard to e.g. fit and ergonomics. Thereby resource consuming physical verifications might be omitted, resulting in faster development and earlier market introduction. Thus accuracy and representability for the target population are key features as product developers come to rely on these models more and more often. Not only for the design itself but also for methods to predict an individual's head shape based on a series of convenient one, two and three-dimensional measurements shape models are useful for parametrized design in a mass-customization production [2], for constructions of true 3D manikins [3] and to assign an individual to its best fitting product size without a trial-and-error fitting.

The world is becoming more and more dependent on computational solutions for the way we work. Where product developers and designers used to do a lot of hands on testing and modeling, a switch was made to digital development [4]. In the past years digital tools and techniques keep on moving further, such that also ergonomics and physical properties are being extensively tested digitally before even the first prototypes are made [5]. These techniques induce the potential to greatly save on development times and costs and this allows for more varying prototypes to be made before selections are made. Online stores are replacing the physical stores as well [6], which implies that near body products and clothing require repeatedly transporting back and forth between the store and customer to try them on. This results in an additional economic and ecological cost that could be eliminated by accurate and representative digital models. A specific example referred to in this study is a model from the human scalp, constructed in [4].

By predicting the user's head shape and digitally analyzing this shape with the 3D models of the (envisioned) product, an optimal fit can be achieved using minimal efforts. These predictions could be done using simple measurements and measurement tools and are easy to accomplish by the users themselves.

The work of Lacko D. et al. [4] has shown that a shape model of a Western population's scalp based on a set of $100 \mathrm{MRI}$ scans is sufficient to predict a human scalp shape with an average error of $1.6+-$ $0.36 \mathrm{~mm}$ using 10 suggested measurements. They also concluded that there is not always the need to measure a large set of measurements to predict this shape, for example by measuring just four simple anthropometric measurements instead of the 10 suggested in their research, the average error increased to just $1.64+-0.39 \mathrm{~mm}$. Although extremes did increase in some areas. When designing products that use a limited set of measurements, these should be chosen accordingly to optimize fit. Thus few and simple measurements could be all that is needed using this statistical shape model to predict accurate 3D shapes of an individual's scalp. The suggested 10 anthropometrical measurements were: head length, face width, Bitragion width, ear height, horizontal ear position, vertical ear position, projected ear height, circumference, arc length and arc width. The shorter list of four contains the head length, head width, circumference and the arc width.

In the research of Lacko D. et al [4] a theoretical verification was performed to calculate the predictions accuracy, but a physical verification of the predicted surfaces was not performed in that experiment. Also the representability of these 100 scans was not tested compared to random samples of the entire population and/or physical people. Because the physical shapes of random test subjects might differ from their predicted ones, this research will verify and thereby validate the use of MRI scans and an amount of 100 samples to generate a shape model that can be used to predict an individual's head shape based on the four convenient measurements described above.

\section{Method}

\section{Overview of the experiment}

In order to analyze the predictive performance of the present shape model with the present four convenient measurements as input value, an experiment was set out to measure specific geometrical characteristics of a series of participants using two methods. First, the four intuitive anthropometrical parameters were measured using simple tools like a tape measure and an outside caliper. Secondly, the 3D locations of a number of points on the participant's heads were determined using a Microscribe $3 \mathrm{D}$ digitizer [5] to obtain a more complex but also more accurate representation of the subject's scalp surface. The simple measurements are used to predict the head shape by the statistical shape model 
constructed in [4] and this shape is then compared in $3 \mathrm{D}$ to the digitized scalp points. Thus the accuracy of the predictions could be verified to the real human's head dimensions. In practice the measurements and the prediction had to be rotated digitally to align their orientation. Euclidean distances from the digitized scalp point to the predicted shape were calculated digitally and the results were compared.

\section{Participants}

For this study a group of 14 participants was selected, consisting of ten males and four females recruited from the staff and students at the faculty of Design Sciences at the University of Antwerp. The subjects were healthy individuals ranging in age from 18 to 55 years old. All 14 participants were chosen randomly and were not included in the MRI database that made up the shape model used in the experiment.

\section{The 4 Intuitive anthropometrical measurements}

To measure the participant's head size in $2 \mathrm{D}$ an outside caliper and a tape measure were used (see figure \#2). The head length (Ophistokranion - Glabella) and the head width (left Preauricular point right Preauricular point) were measured using the caliper, and the head circumference (over the Ophistokranion and Glabella) and arc width over the head (from left Preauricular point to right Preauricular point) using the tape measure (see table \#1 for detailed anthropometrical definitions). All measurements were taken twice, and where the difference was more than $2 \mathrm{~mm}$, measured twice again. The averages of these two repetitions were then taken for each measurement, resulting in four measurements in total. The measurements taken with the caliper were transferred to a ruler and thus recorded since the outside caliper itself has no scale.

Table 1: Overview of the one and two-dimensional measurements used.

\begin{tabular}{|c|c|}
\hline Head length & From the Ophistokranion to the Glabella \\
\hline Head width & From the left-Preauricular point to the right-Preauricular point \\
\hline Head circumference & Around the head through the Ophistokranion and the Glabella \\
\hline $\begin{array}{l}\text { Arc length over the width of } \\
\text { the head }\end{array}$ & $\begin{array}{l}\text { From Preauricular point to Preauricular point perpendicular to the } \\
\text { transversal plane }\end{array}$ \\
\hline
\end{tabular}

These measurements are the most intuitive since all points are easily recognizable and easy to explain [9].

The Glabella is defined as the point in between the eyebrows and above the nose.

The Ophistokranion is easiest to find using the caliper and searching the furthest distance from the Glabella.

The Preauricular point is best definable as the point in the dent between the upper edge of the Tragus and the Daith. This point can be found on both the left and the right ear.

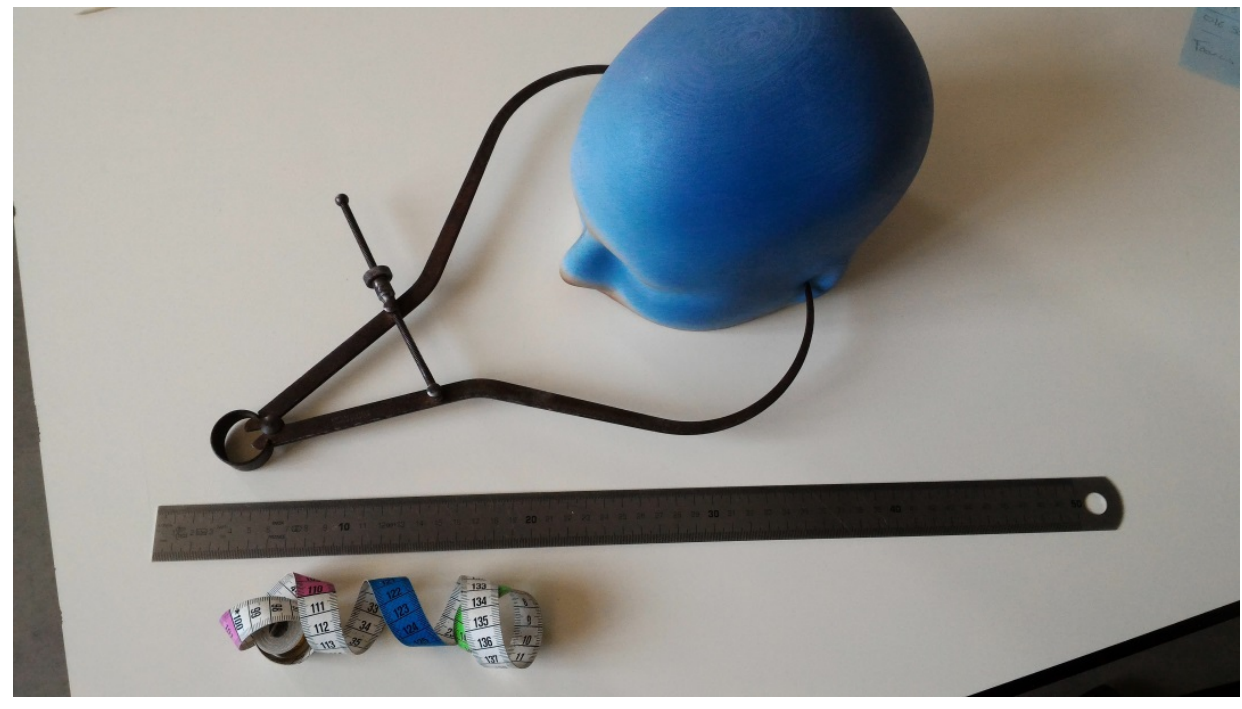

Fig 2: Measuring tools used: Outside caliper, long ruler and a tape measure 


\section{Head shape prediction}

These four measurements were used to feed the shape model prediction tool (more details of this can be found in the paper of Lacko D. et al [4]) which returns an .stl file (see figure 1) containing the predicted shape with those measurements. The prediction is done by a custom Python code of which a simplified online tool version will become available online soon [6]. In short it searches for the most likely shape of the head for a person with those measurements entered. This shape is not one of the 100 shapes in the shape model but a blend of all of them that matches as good as possible the statistical most likely shape for those entered measurements.

\section{$3 D$ reference measurements}

Next the 3D shape of the participant's head was determined by digitizing the 3D locations of a set of points on the scalp using a Microscribe 3D digitizer [5]. First three reference points were digitized: the Glabella and both Preauricular points, and then a series of 38 points following a custom EEG cap [11](see figure \#3) based on the 10-20 system. Some extra points had already added to the basic 32 locations and the holes were enlarged so the measuring probe could fit easily. The sharp tip of the measuring probe was replaced with a plastic flat tip of $4 \mathrm{~mm}$ to enhance the physical comfort for participants (see figure \#4). These measurements were stored in a .txt file containing the $x-y-z$ coordinates of each location in order.

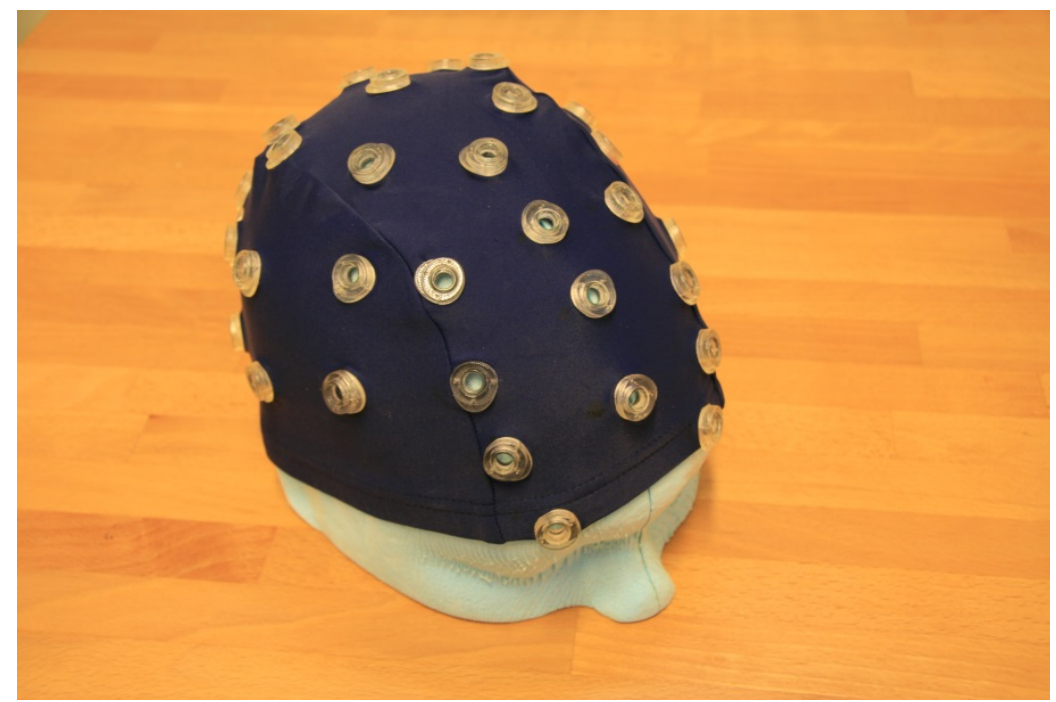

Fig 3: Custom EEG cap used to locate the reference locations.

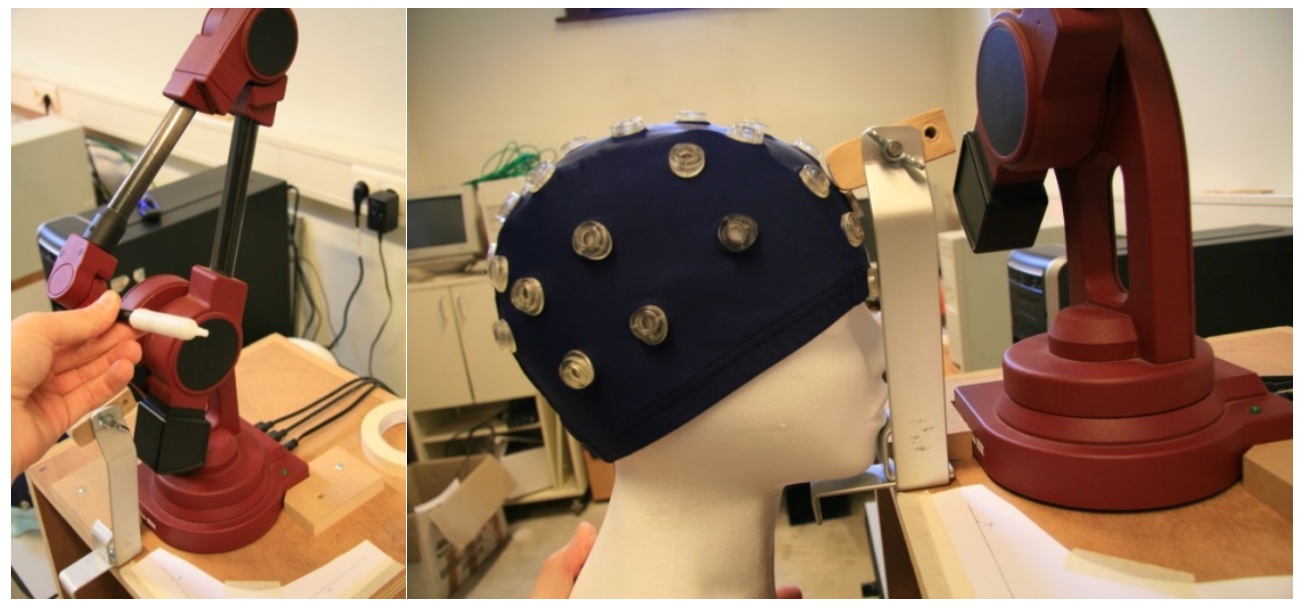

Fig 4: Measuring setup with the digitizer, the head fixture and cap on a (smaller than regular) foam head model.

A 3D digitizing probe was chosen rather than a 3D scanner because these points have greater accuracy $(0.05 \mathrm{~mm}[8]$ versus $0.5 \mathrm{~mm})$ and the probe reaches down to the skin layer rather than a scanner which would scan the hair layer. Otherwise, further image processing or shape compensation would be necessary in order to get to the actual skin layer's shape. 


\section{Data handling}

The data was rotated by a custom made script that aligned the three reference locations of the measurements (Glabella and both Preauricular points) to the corresponding locations of these points on the predicted head surface in the .stl file. This was a preliminary alignment to make sure the orientation of the head was already close to the desired location. A second alignment was then performed with an iterative closest point (ICP) implementation from VTK [6]. This performed a more subtle re-alignment only rotating by a few degrees at most to align the data points more precise to the predicted surface. Otherwise a small error whilst measuring the three reference points would lead to a large error in the alignment.

Rotation of the data points was performed by calculating the center between the Glabella and both Preauricular points for both the predicted shape and the 38 measurements. All datasets were moved such that the centers of all coincided with the origin $(0,0,0)$. Then the angle of the line between both Preauricular points was calculated compared to the reference axis and rotated in order to make sure the line between both Preauricular points collinear to the axis. These rotations were performed using trigonometric functions as well as the angle measurements. The same procedure was then repeated to align the Glabella to center line to its reference axis (see figure \#5).

The iterative closest point method that performed the detailed alignment compared all measurement points and searched for the closest match to the prediction surface by calculating the cumulative distances for all points. The ICP method was constrained to a maximum of 1000 iterations and rotation was measured again after the process to make sure no extreme cases (local optima) were used for alignment (which in some cases resulted in the points being aligned to the back of the head instead of to the top).

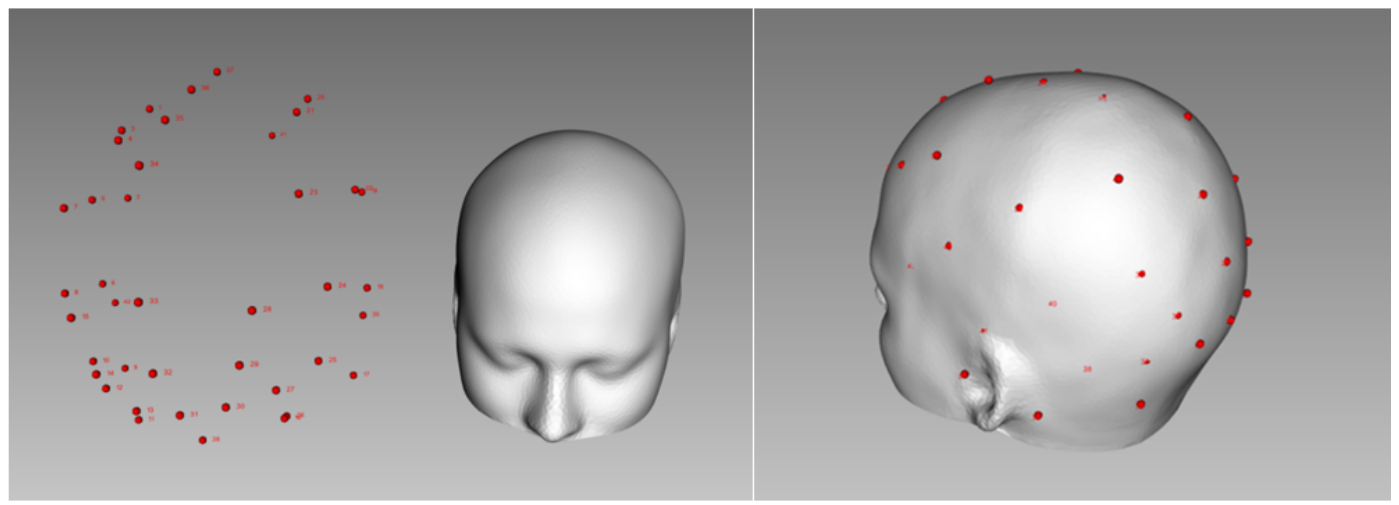

Fig 5: Raw and aligned data: Measurements plotted on the corresponding .stl file

\section{Difference measurement}

Finally the Euclidean distance between the measurement points and the predicted surface were measured using an intersection method. This plots a line from the measured point to the center of the shape. On this line the intersection on the predicted shape is pinpointed, and the distance between the intersection and the original scalp location is recorded. This method gives the best measurements since perpendicular measurements to the predicted shape could give a bigger measurement error. In order to gain more accurate measurements the predicted shapes would need to be smoothed to a curved surface first instead of a triangle mesh the prediction consists of. A visual representation of these points can also be plotted on a sample predicted shape (see figure \#5).

\section{Data processing}

The final data consists of an array of 1D Euclidean distances from the measurement point to the predicted shape for all 38 measurements and for all of the 14 participants. These distances have a sign indicating if the prediction was either too large or too small for that measurement point, i.e. of the scalp point lies within or outside the predicted surface. For analysis both these values and their absolute values were processed separately. The signed values give an insight in the average error but balance each other out towards 0 since part of the measurements are negative. On the contrary, the absolute values provide better insight in the average error. Thus the data is analyzed by taking averages and the standard deviations of both types of data. 


\section{Results}

The average error of the prediction compared to the 3D measurements of the test subjects is $2.12 \mathrm{~mm}$ with a std. of $1.87 \mathrm{~mm}$. Local extremes range from $0 \mathrm{~mm}$ to $9.37 \mathrm{~mm}$ on the worst measurement and prediction combination. The simplified data overview per subject can be seen in table \#2.

Table 2: Overview of absoluted measured data per test subject.

\begin{tabular}{l|llll|} 
Subject & Avg.(mm) & Min. $(\mathrm{mm})$ & Max. $(\mathrm{mm})$ & Std. $(\mathrm{mm})$ \\
\hline 1 & 2,76 & 0,03 & 9,37 & 2,23 \\
2 & 2,13 & 0,01 & 8,21 & 1,95 \\
3 & 1,80 & 0,04 & 5,90 & 1,34 \\
4 & 1,93 & 0,05 & 6,28 & 1,46 \\
5 & 2,03 & 0,01 & 5,90 & 1,59 \\
6 & 1,92 & 0,01 & 5,99 & 1,55 \\
7 & 3,09 & 0,01 & 8,85 & 2,18 \\
8 & 1,48 & 0,00 & 4,43 & 1,19 \\
9 & 1,11 & 0,03 & 3,63 & 0,80 \\
10 & 1,52 & 0,02 & 5,02 & 1,06 \\
11 & 3,34 & 0,00 & 9,36 & 2,75 \\
12 & 1,81 & 0,04 & 7,07 & 1,79 \\
13 & 3,03 & 0,02 & 8,97 & 2,40 \\
14 & 2,11 & 0,03 & 6,79 & 1,69 \\
\hline Total metrics: & 2,12 & 0,00 & 9,37 & 1,87 \\
\cline { 2 - 5 } & Avg.(mm) & Min. (mm) & Max. (mm) & Std. (mm) \\
\cline { 2 - 5 } & & & &
\end{tabular}

The signed values average to an error of $-0.82 \mathrm{~mm}$ and a std. of $3.42 \mathrm{~mm}$. In other words, the predicted shapes are slightly larger than the measurements, but this could be explained due to impression of the skin layer by the probe. The deviation is larger than that of the absolute values since the error is now in both directions.

Arguably the measurements taken with the digitizer still contain a measurement error. The participants were instructed to keep their head still in a fixture, but small movements couldn't be fully prevented since their entire head had to be reachable and within range of the digitizer. Also the measuring probe was remade to a diameter of $2 \mathrm{~mm}$ thus to a bit more comfortable for the participants, which also means more hair could remain between the probe and the skin layer.

Since all digitizer measurements were also performed twice per subject, a value can also be placed on the accuracy of the measurements taken. This difference in the two measurements each were about $0.76 \mathrm{~mm}$ on average with a std. of $0.62 \mathrm{~mm}$ and thus within a reasonable range.

\section{Discussion}

Further research should prove the merits of statistical shape models for real life products [13] but should also investigate the effect of hair on both the predictions as the presence of hair might greatly influence the fit of products for their target population. Knowing the thickness of the hair is important for both the usefulness of the shape models as for the development of products for the users since most of the head worn products will need to fit on top of the hair instead of on the skin layer (notable exception being: Brain computer interfaces).

Also, a recommendation should be made here that when choosing the measurements that are used in the products design these should be chosen in such a way as to minimize possible prediction errors. While the measurements that were used to create the shape predictions will be accurate, other measurements can still vary by a larger margin. For example, for a prediction on the basis of the head circumference, the predicted shape's circumference will exactly match the input circumference value, but measurements like ear height will be less accurate. A tool should be made that visualizes the possible error on each part of the shape. Such a tool is theoretically possible, since all shape data is contained in the shape model.

The power of the prediction tools like used in this study is not only limited to delivering a predicted shape for a set of measurements but can also be used to analyze the size and shape of the population, which is useful not only for research projects but certainly for product design and development where better fit can be achieved. 
In products where mass-customization is the desired production method, shape models can also help in one of two ways; It can either provide an adapting shape to work on, so that a model designed on an average measurement can be adapted to an individual's dimensions, or it could just as well be used to create a parametric design of the final product itself, depending on the product and the production methods.

But designers should be cautious since the local extreme errors of up to $9.37 \mathrm{~mm}$ (see table \#2) should possibly still ought to fit the design (they could just as well be wrong predictions as they could be deformations on the user's head shape witch should still be accompanied for up to a certain margin).

\section{Conclusions}

In this experiment, the scalp shapes of 14 participants were predicted using a shape model on the basis of four simple measurements: head length, head width, head circumference and the arc length over the width of the head. The predicted shapes were compared to the actual head shapes by digitizing the position of 38 points on the participant's head and calculating the Euclidian distance between these points and the predicted surfaces. The physical error measured is a bit larger $(2.12$ $\mathrm{mm}$ ) then the theoretical error determined in previous research $(1.64 \mathrm{~mm})$, but within acceptable range. The standard deviation has increased more from 0.39 to $1.87 \mathrm{~mm}$. These results indicate that statistical shape models are not only usable in theory but are now also verified in a physical setup.

This means products can be designed using these shape model in order to either supply a reference model for design or even to adapt a mass-customization process were products could be made to fit using only 4 easy to measure measurements.

\section{References}

[1] R. Ball, "3-D Design Tools from the SizeChina Project.," Ergonomics in Design: The Quarterly of Human Factors Applications, vol. 17, no. 3, pp. 8-13, 2009.

[2] F. Piller, "Mass Customization: Reflections on the State of the Concept.," International Journal of Flexible Manufacturing Systems, vol. 16, no. 4, pp. 313-334, 2004.

[3] D. Lacko et al, "An Anthropometric Shape Model for the Design of Sports Helmets," in European Conference on Protective Clothing (ECPC), Bruges, 2014.

[4] C. Shu en et al, „Geometry Processing and Statistical Shape Analysis of,” in 17th World congress on Ergonomics, CNRC, 2009.

[5] e. a. Chih-Hsing Chu, „Design Customization of Respiratory Mask based on 3D,” INTERNATIONAL JOURNAL OF PRECISION ENGINEERING AND MANUFACTURING , vol. 16, nr. 3, pp. 487-494, 2015.

[6] S. Bellman en et al, „Predictors of online buying behavior,” Communications of the ACM, vol. 42, nr. 12, pp. 32-38, 1999.

[7] D. Lacko et al, "Evaluation of an anthropometric shape model of the human scalp," Applied Ergonomics, vol. 48, pp. 70-85, 2015.

[8] Microscribe, „Microscribe MX 3D Digitizer,” Microscribe, 2015. [Online]. Available: 3d-microscribe.com. [Geopend 29 april 2015].

[9] B. Oetteking, Lehrbuch der Antropologie, in Systematischer Darstellung, Stutgart: American Anthropologist, 1957.

[10] J. Vleugels, "Cadans online tool," Cadans, 2015. [Online]. Available: http://cadans.uantwerpen.be.

[11] Trans Cranial Technologies, "10/20 System Positioning," MedCaT Supplies, 2015. [Online]. Available: www.trans-cranial.com. [Accessed 30 April 2015].

[12] VTK, "VTK: The Visualization Toolkit," Kitware, 2015. [Online]. Available: http://www.vtk.org/. [Accessed 25 May 2015].

[13] S. Alemany en et al, „A multidimensional approach to the generation of helmets' design criteria: a preliminar study," Work, vol. 41, nr. 1, pp. 4031-4037, 2012. 